\title{
Temporal Control of Movements in Sensorimotor Synchronization
}

\author{
Gisa Aschersleben \\ Max Planck Institute for Psychological Research, Munich, Germany
}

Published online August 17, 2001

\begin{abstract}
Under conditions in which the temporal structure of events (e.g., a sequence of tones) is predictable, performing movements in synchrony with this sequence of events (e.g., dancing) is an easy task. A rather simplified version of this task is studied in the sensorimotor synchronization paradigm. Participants are instructed to synchronize their finger taps with an isochronous sequence of signals (e.g., clicks). Although this is an easy task, a systematic error is observed: Taps usually precede clicks by several tens of milliseconds. Different models have been proposed to account for this effect ("negative asynchrony" or "synchronization error"). One group of explanations is based on the idea that synchrony is established at the level of central representations (and not at the level of external events), and that the timing of an action is determined by the (anticipated) action effect. These assumptions are tested by manipulating the amount of sensory feedback available from the tap as well as its temporal characteristics. This article presents an overview of these representational models and the empirical evidence supporting them. It also discusses other accounts briefly in the light of further evidence. (c) 2001 Elsevier Science
\end{abstract}

The timing of actions can be analyzed by studying simple repetitive tasks in which participants are required to accompany a predictable stimulus with a simple movement. One such task is the synchronization task: Participants use, for example, their right index finger to tap on a key at a given rate (tapping task) with the beat being presented by a metronome. Performance on such synchronization tasks has been studied since Stevens in 1886 (see Fig. 1).

Stevens, however, used the synchronization phase to establish a sequence of regular key presses. After some signals, the metronome was switched off and participants had to continue tapping at the same rate. He then examined regularity of the taps in this so-called continuation phase. Hence, such studies have analyzed mainly the size and variability of intertap intervals (see, for overviews, Miedreich, 2000; Vorberg \& Wing, 1996).

However, in the present context, we focus on the synchronization phase, and ask how precisely the tap can be timed relative to the click. The dependent variable in which we are then interested is the interval between the touch of the key and the presentation of the pacing signal. Although people think this task will be rather easy

This research was supported partially by a grant from the Deutsche Forschungsgemeinschaft (SFB 462-99). I thank Jeff Summers and Ralf Krampe for helpful criticisms, suggestions, and comments on an earlier draft as well as Jonathan Harrow for suggestions on English style.

Correspondence concerning this article and requests for reprints should be addressed to the author who is at the Max-Planck-Institut für Psychologische Forschung, Postfach 340121, D-80098 München, Germany. E-mail: aschersleben@mpipf-muenchen.mpg.de or http://www.mpipf-muenchen.mpg.de/ raschersleben. 


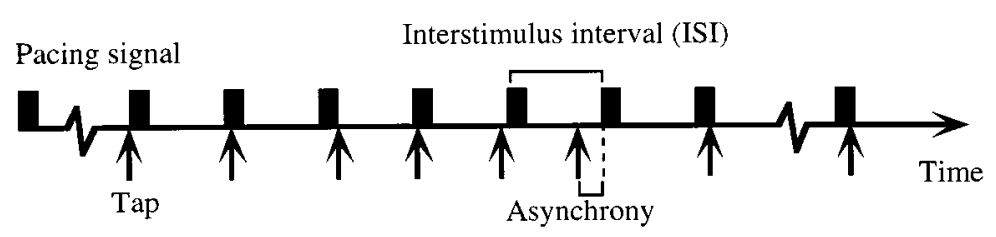

FIG. 1. The synchronization task.

and simple, a systematic error that is called negative asynchrony is observed. This means that the tap precedes the click by about 20 to $80 \mathrm{~ms}$. This effect was described already more than 100 years ago (e.g., Dunlap, 1910; Johnson, 1898; Miyake, 1902) and has been replicated in a number of studies (e.g., Aschersleben \& Prinz, 1995, 1997; Fraisse, 1980; Kolers \& Brewster, 1985; Mates, Müller, Radil, \& Pöppel, 1994; Repp, 2000; Thaut, Tian, Azimi-Sadjadi, 1998; Vos, Mates, \& van Kruysbergen, 1995, Wohlschläger \& Koch, 2000).

Although the size of this asynchrony depends to a great extent on the experimental conditions under study, there are large interindividual differences as well. Whereas the mean asynchrony observed in a specific person may be very small and even close to zero (positive asynchronies are rarely observed), persons exhibiting negative asynchronies in the order of $100 \mathrm{~ms}$ in hand tapping without being aware of this huge anticipatory tendency are found repeatedly. One factor influencing the individual size of the negative asynchrony is the way in which the tap is performed. As we shall discuss in greater detail below, the amplitude of the finger movement and the force applied to the key exert a strong influence on the size of the negative asynchrony, and persons probably differ in their movement patterns. Another important factor is musical experience. Musically untrained persons exhibit an asynchrony that is, on average, $10 \mathrm{~ms}$ larger than that of persons who report playing an instrument as a hobby (Aschersleben, 1994). The impact of musical experience can be seen even more clearly in highly trained musicians. When students at a music academy were asked to synchronize their taps to an auditory pacing signal, the mean asynchrony was even smaller $(-14 \mathrm{~ms}$ vs -40 to $-50 \mathrm{~ms}$ in untrained persons). Nonetheless, it is important to note that each single participant exhibited a negative asynchrony; that is, nobody tapped in exact synchrony or with a delay (Ludwig, 1992). The mean asynchrony is even smaller in professional musicians (e.g., pianists), who, occasionally, are able to tap in exact synchrony (see, e.g., Repp, 1999).

Experiments on so-called pseudo-synchronization indicate that the synchronization error is in no way an artifact of the experimental situation but seems to be necessary for persons to gain the subjective impression of being in synchrony. Participants in such experiments start tapping to a metronome. Without being informed about the change, the auditory pacing signal is turned off after some beats and replaced by an auditory feedback signal. In other words, each time the finger touches the key, an auditory signal is presented (with the same intensity and frequency as the former pacing signal). Thus, participants tap in exact synchrony to what seems to be the pacing signal (although, in fact, it is the feedback from their own taps). The consequence is that they speed up their taps (Fraisse \& Voillaume, 1971; Vos, Helsper, \& van Kruysbergen, 1992). This can be interpreted as a tendency to reestablish the negative asynchrony between tap and click because the physical synchrony is not perceived as such.

To analyze the influence of training on asynchrony, Aschersleben (2000a, 2000b) asked participants to tap for 10 sessions (about 10,000 taps in total) under different conditions. If participants just tap without gaining any informative feedback about 
their performance, no change in asynchrony is observed. It is only when they are informed after each tap about the size and the direction of the asynchrony (knowledge of results) that participants are able to tap in exact physical synchrony after 10 sessions. However, under these conditions, participants report that they have to delay their tap subjectively, that is, to tap too late to produce the required objective synchrony.

\section{AN EXPLANATORY ACCOUNT: THE ROLE OF SENSORY FEEDBACK}

Although the anticipatory error has been known for more than 100 years, the underlying mechanisms are still not understood completely. The literature proposes a number of accounts that place the cause for the anticipatory error at different levels of processing. Some accounts assume that synchrony between tap and pacing signal has to be established at the central representation level in order to be perceived as being in synchrony. Other models are based on the assumption that our cognitive system refers to the physical events. The present article focuses on the first class of models; the other accounts will be discussed briefly in the second part of this article.

Recent accounts suggest that synchrony is not only controlled by but also established at a central representational level on which both stimuli and actions are represented in terms of their sensory consequences (Aschersleben, Gehrke, \& Prinz, in press a; Aschersleben \& Prinz, 1995, 1997; Gehrke, 1996; Prinz, 1990, 1997). Therefore, action control in synchronization tasks is concerned less with the temporal relationship between the auditory input and the motor output, and more with achieving synchrony between perceived click and perceived tap. ${ }^{1}$ If this is the case, the time it takes to perceive the click and the tap becomes crucial, particularly as auditory and kinesthetic-tactile stimulation can be assumed to differ in processing speed. In fact, as the temporal delay between actual and perceived click is likely to be shorter than the delay between actual and perceived tap, the actual tap must precede the actual click to achieve synchrony between the perceived events at a central level, thus producing the observed negative asynchrony between click onset and overt tap.

Therefore, the representational accounts are based on two assumptions. (a) Central representations of click and tap are made to coincide; and (b) the central representation of the tap is based on somatosensory feedback from the movement of the finger (and not on the motor command). These assumptions have been used to develop two hypotheses that differ in the way they localize the cause of the asynchrony. According to the nerve-conduction hypothesis (Paillard-Fraisse hypothesis), the anticipatory error is due to differences in the nerve conduction time between click and tap and their corresponding central representations (Aschersleben, 1994; Aschersleben \& Prinz, 1995, 1997; Fraisse, 1980; Paillard, 1949). In contrast, the sensory accumulator model (Aschersleben et al., in press a; Gehrke, 1996) focuses on processes at a central level as the main cause for the effect.

\section{The Nerve-Conduction Hypothesis}

Unlike the sensory accumulator model, the nerve conduction hypothesis assumes that the negative asynchrony has its origins in peripheral processes. Because it takes more time for the sensory information from the tip of the finger (resulting from the tactile and kinesthetic feedback from the tap) to travel to the brain than that from

\footnotetext{
${ }^{1}$ This approach relates closely to the idea of looking at timing from the perspective of behavioral goals that Drewing and Aschersleben (2001) have proposed to account for the "bimanual advantage", in continuation tapping.
} 


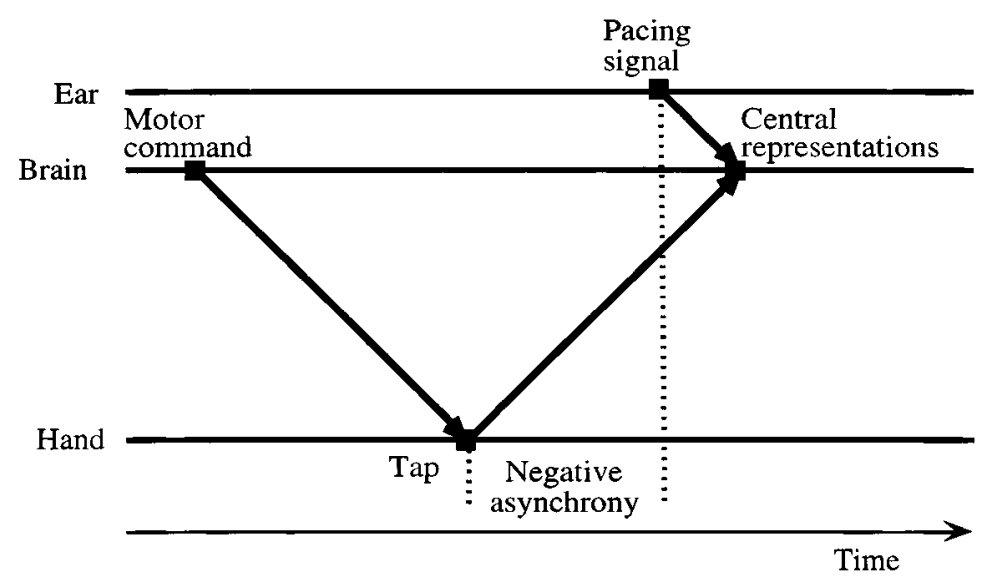

FIG. 2. The nerve-conduction hypotheses (based on Prinz, 1992).

the ear (resulting from the auditory pacing signal), the tap has to precede the click to establish synchrony at the level of central representations (Aschersleben \& Prinz, 1995, 1997; Fraisse, 1980; Paillard, 1949; see Fig. 2).

One obvious way to test this assumption is to manipulate the feedback coming from the tap or, more precisely, the temporal delay between actual and perceived tap. The longer this delay, the longer the actual tap must lead over the actual click to achieve coincidence between the two perceived events, and the more pronounced the negative asynchrony should be. In fact, several experimental manipulations have provided empirical support for this prediction. Experiments designed to test these predictions follow the principle of manipulating the transduction delays between the tap and its central representation described above. The manipulations have been to delay the intrinsic feedback, add extra feedback components, and delay this external feedback.

A simple way to manipulate the transduction delay between the tap and its central representation is to study foot tapping. Participants are asked to tap with different effectors, such as hand or foot, under the assumption that the temporal delay between actual and perceived tap would increase with the "neural distance" between effector and brain. As expected, the anticipatory error was more pronounced with foot than with hand tapping, and this effect, which is rather substantial (45 ms) under controlled feedback conditions (eliminating visual and auditory feedback from the tap), was independent of the body side involved and of whether the movement was performed with one effector or two effectors simultaneously (Aschersleben \& Prinz, 1995; Billon, Bard, Fleury, Blouin, \& Teasdale, 1996; Fraisse, 1980).

Further evidence supporting the nerve-conduction hypothesis comes from experiments on self-initiated tapping with hand and foot. Participants were asked to produce synchronized finger and foot taps without any pacing signal. Here as well, a lead could be found for foot tapping (Bard, Paillard, Teasdale, Fleury, \& Lajoie, 1991; Bard et al., 1992; Billon et al., 1996; Paillard, 1949; Stenneken, Aschersleben, Cole, \& Prinz, in press).

The influence of additional auditory feedback on the timing of the tap has been studied by presenting an auditory signal each time the participant touches the key. Assuming that the timing of the tap is not dependent on any single feedback component but on an integrated percept of all feedback components ("joint event code") being available at a central level, a reduced anticipatory error could be expected (Aschersleben \& Prinz, 1995, 1997; see, for a similar assumption, Fraisse, Oléron, \& 
Paillard, 1958). In the case of additional auditory feedback, the tap would be represented by a late internal (tactile/kinesthetic) component and an early external (auditory) component. Any integration of these two parts should lead to a reduced negative asynchrony. This actually could be demonstrated in a number of experiments (e.g., Aschersleben \& Prinz, 1995, 1997; Mates \& Aschersleben, 2000; Mates, Radil, \& Pöppel, 1992; O’Boyle \& Clarke, 1996).

Additional support for the assumption of a joint event code can be found in studies on delayed auditory feedback. These experiments introduced a delay between the touch of the key and the presentation of the corresponding feedback tone. When this delay was so small that participants were not aware of the experimental manipulation (less than $100 \mathrm{~ms}$ ), an increase in asynchrony was observed with increasing delay (Aschersleben \& Prinz, 1997, Mates \& Aschersleben, 2000). Moreover, the assumption that a joint event code arises from a linear combination of the tactile/kinesthetic and the auditory feedback component would lead us to expect a linear relationship between asynchrony and delay. Corresponding experiments confirmed this assumption through the slope of the regression function (which was -.4), indicating that the contribution of the auditory feedback signal is somewhat weaker than that of the tactile/kinesthetic signal (Aschersleben \& Prinz, 1997).

\section{The Sensory Accumulator Model}

The sensory accumulator model is based on the same main assumptions as the nerve conduction hypothesis, that is, synchrony is established at the level of central representations, and the tap is represented by its somatosensory feedback. However, whereas the nerve-conduction hypothesis stresses the role of delays derived from conduction times along afferent pathways, the sensory accumulator model assumes that the processing times needed to generate a central representation of peripheral events may also play a role in the observed asynchrony. The model assumes that for an external event to be experienced and timed, its central representation has to be experienced as one neural entity. The processing times necessary for generating this neural state would be threshold-dependent, and this would change according to the density of afferent neural signals generated by the physical events. Hence, the model is based on the assumption of an accumulation function whose steepness determines the time elapsed between an external event and its central representation. Consequently, as the auditory pacing signal used in the synchronization task has a steeper accumulation function than the tap, a negative asynchrony obviously has to be expected (Aschersleben et al., in press a; Gehrke, 1995, 1996; see Fig. 3). One relevant

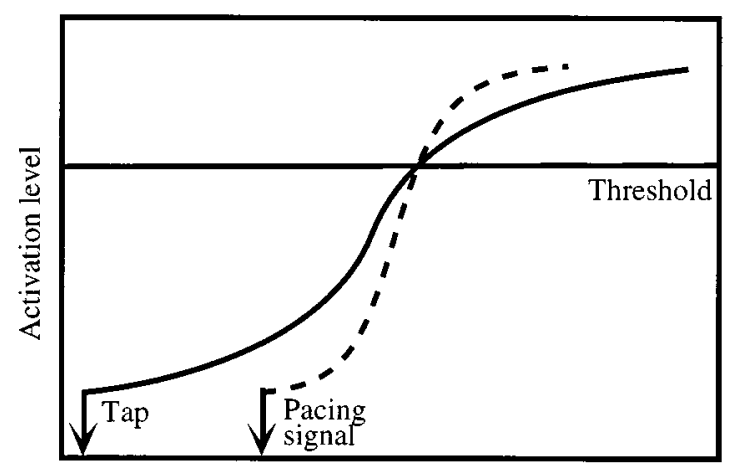

Time

FIG. 3. The sensory accumulator model (based on Gehrke, 1996). 
factor influencing the size of the anticipatory error would then be the density of afferent signals arriving at a central level: the more afferent signals (in a unit of time), the earlier a threshold should be reached.

First of all, it is necessary to demonstrate whether this model is able to account for the empirical evidence gathered in favor of the nerve-conduction hypothesis. The increased asynchrony in foot tapping compared with hand tapping could be explained by the fact that the density of receptors at the toe is much lower than that at the tip of the finger. Correspondingly, the number of afferent signals from the former that reaches the central level within a given time interval is much lower. This should lead to a more gradual accumulation function and, therefore, to a larger asynchrony in foot tapping conditions.

When considering the influence of additional auditory feedback, the sensory accumulator model makes the same predictions as the nerve-conduction hypothesis. As with the auditory pacing signal, a steeper accumulation function is postulated for the auditory feedback signal compared with the tactile/kinesthetic feedback component. Because the tap is represented centrally by a single ( joint) event code composed of all available components, this event code is dated earlier (because of the steeper auditory component), thus resulting in a reduced asynchrony. Correspondingly, when the additional auditory feedback is delayed, the timing of the joint event code is delayed, leading to an increase in asynchrony.

In addition to these post hoc explanations of available evidence, the sensory accumulator model makes further predictions that have been tested with experiments in which the main manipulations are applied to the internal feedback components. One prediction of the accumulator model is that intensifying feedback from the finger should reduce the time required to establish the central representation of the tap, and, as a consequence, reduce the asynchrony. One possible way of testing this prediction is to manipulate the maximum amplitude of the finger movement. A large amplitude accompanies an increase in force and, thus, an increase in tactile stimulation. In addition, larger finger movements are performed with higher velocity, which, therefore, increases kinesthetic feedback. Altogether, a large finger amplitude intensifies sensory stimulation at the finger, which should result in a reduced asynchrony. Indeed, when participants produce large amplitudes (e.g., $7 \mathrm{~cm}$ ), the corresponding asynchrony $(-25 \mathrm{~ms})$ is significantly smaller than under conditions with small finger amplitudes $(1 \mathrm{~cm}$; corresponding asynchrony $-60 \mathrm{~ms}$; Aschersleben et al., in press a; Gehrke, 1996).

The most direct way to test the hypothesis that sensory feedback is an important factor determining the timing of actions is to eliminate it completely. There are at least two possible ways of doing this: One is to remove sensory feedback temporarily in healthy people through local anesthesia and the other, to study patients with loss of sensory feedback. In both kinds of testing, it is important to make sure that the transfer of the motor command (i.e., via efferent nerves) is unimpaired.

Aschersleben and colleagues (Aschersleben, Gehrke, \& Prinz, 2001) studied the influence of local anesthesia to the right index finger on the timing of taps. Anesthetizing the finger performing the tapping movement suppressed tactile reafferent information without disturbing the reafferent discharge of the joint and muscle receptors. Under these conditions, neither the maximum tapping rate nor synchronized tapping without making contact with the key (tapping in the air like a conductor) showed any influence of peripheral nerve block. This result indicates that both the efferent fibers and the transmission of kinesthetic feedback information were impaired. However, in the usual finger tapping task, the asynchrony increased significantly. First, this result clearly demonstrates the important influence of tactile feedback on the timing of the taps. Second, the increased asynchrony can be interpreted as empirical 
support in favor of the sensory accumulator model. This model assumes a steeper accumulation function for the tactile compared with the kinesthetic part of the finger tap. Thus, in the usual tapping task, a joint event code consisting of an early tactile and a late kinesthetic part is brought to coincide with the pacing signal. By eliminating the (early) tactile part via local anesthesia, the timing of the tap should be delayed at a central level, thus, resulting in an increase in the anticipatory error. This is exactly what the data showed.

Patients who meet the criterion of a loss of sensory feedback with no impairment to efferent fibers are hard to find because of the infrequency of this condition. Bard and colleagues (Bard et al., 1992) studied a patient who was almost completely deafferented (see, for a description, Cooke, Brown, Forget, \& Lamarre, 1985). In a simple reaction time task, this patient showed behavior similar to that of healthy controls. Her performance differed, however, under conditions of self-initiated hand and foot tapping. As already indicated above, a lead of the foot tap can be observed in healthy participants, supporting the idea of synchronized afferent feedback. However, the patient did not show this predating of the foot but produced results similar to those on the reaction time task.

Further support for the notion that sensory feedback plays an important role in the timing of repetitive movements comes from a recent study with another deafferented patient. This patient was suffering from a purely sensory neuropathy leading to a total loss of kinesthetic and tactile sensitivity for the whole body below the neck while leaving the motor system intact (see, for a description, Cole, \& Sedgwick, 1992). A synchronization task manipulated the amount of (extrinsic) sensory feedback from the tap. Results indicated clearly that the presentation of feedback had a strong impact on the performance of the deafferented patient by considerably improving his performance (Aschersleben, Stenneken, Cole, \& Prinz, in press b).

\section{Summary of the Role of Sensory Feedback}

The general account of negative asynchrony presented above assumes that synchrony of events is established at the central level on which the corresponding events tap and pacing signal are represented via their sensory consequences. According to this model, the negative asynchrony arises because of differences in the peripheral and/or central processing time of the tap compared with the click.

Two concrete hypotheses are presented: the nerve-conduction hypothesis, stressing the importance of peripheral processes; and the sensory accumulator model, localizing the cause of the negative asynchrony at a central level. Empirical tests of both hypotheses are based on the manipulation of internal and external feedback. Delayed intrinsic feedback (foot tapping) leads to an increase in asynchrony, whereas adding external feedback (auditory feedback) results in a reduction of the anticipatory error. Furthermore, intensifying internal feedback (increased force) leads to reduced asynchrony, and eliminating internal feedback (local anesthesia) increases the error. In sum, by manipulating the time between the tap and its central representation, the amount of negative asynchrony can be influenced in a predictable manner, thus providing empirical support for the importance of sensory action effects in the timing of events.

\section{FURTHER EVIDENCE AND ADDITIONAL ACCOUNTS}

The above overview indicates that there is ample evidence for the importance of sensory action effects on the timing of actions. However, further evidence on the synchronization paradigm makes it clear that additional factors must be involved in negative asynchrony. The following presents an overview of this evidence and a brief discussion of the hypotheses proposed to explain it. 


\section{Manipulating Features of the Pacing Signal}

A number of studies are described in the literature that manipulate features of the pacing signal such as its modality (auditory, visual, tactile), its duration, the interstimulus interval, or the structure of the (usually empty) interstimulus interval. Usually, auditory stimuli are presented as the pacing signal in sensorimotor synchronization tasks. This is because the perception of temporal structures is much better in the auditory than the visual modality (the reverse is true for the perception of spatial structures). The few studies that have examined visual or tactile pacing signals reveal contradictory results. Kolers and Brewster (1985) report a reduction in asynchrony under conditions applying visual signals rather than clicks (and even positive asynchronies under conditions with very short intertap intervals), thus replicating earlier studies (Dunlap, 1910; Fraisse, 1948; Miyake, 1902).

Tactile pacing conditions (using a brief mechanical stimulation of the left index finger as pacing signal) also seem to produce a smaller asynchrony than auditory signals. In Kolers and Brewster's (1985) study, the asynchrony for tactile pacing took an intermediate size between those for visual and auditory pacing. However, Müller et al. (2001) observed no asynchrony at all under conditions with tactile pacing. That is, participants were able to synchronize their taps exactly with the pacing signal; interestingly, not only under conditions with tactile pacing at the finger but also with tactile pacing at the toe.

This finding led Müller et al. (2001) to assume the existence of modality-specific temporal integration zones within which events are perceived as being simultaneous. If both the tap and the pacing signal are in the same (tactile) modality, both integration zones are of equal size, and no asynchrony arises. However, the integration zone for the auditory modality is assumed to be smaller (because of the higher temporal resolution) than that for the tactile modality, thus, leading to negative asynchrony. A similar interpretation applies to conditions with a visual pacing signal.

Another important feature of the pacing signal that seems to exert an influence on the size of negative asynchrony is its duration. Usually, this is rather short (up to 15 ms). Vos and co-workers (Vos et al., 1995) manipulated the signal duration systematically between 1 and $300 \mathrm{~ms}$. They observed a decrease in asynchrony with increasing duration, and explained this effect with the P-center hypothesis (e.g., Morton, Marcus, \& Frankish, 1976). This assumes that each event that is extended over time has a perceptual center ( $\mathrm{p}$-center) that differs from the onset of the stimulus. Its position in time is assumed to depend on, among others, stimulus duration; that is, with increasing duration, there is an increasing delay between stimulus onset and p-center. According to the synchronization paradigm, it is not the onset but the p-center of the pacing signal that is synchronized with the tap, thus leading to the observed asynchrony between tap onset and click onset.

Additional support for the important role of a perceptual center in temporally extended pacing signals can be found in studies by Auxiette (e.g., Auxiette, 1992; Auxiette \& Boucart, 1995). When she presented syllables instead of clicks as the pacing signal in a synchronization task, she even observed positive asynchronies. She interpreted these results as indicating that the perceptual center of the syllable (and not its onset) is synchronized with the tap. Correspondingly, her perceptual experiments revealed that the syllable had to be presented before a tactile stimulus if both were to be perceived as being simultaneous. ${ }^{2}$

\footnotetext{
${ }^{2}$ Aschersleben (1994) tested the validity of one version of the p-center hypothesis on the tap side by analyzing correlations between tap duration and asynchrony (under the assumption that increasing tap duration should delay the p-center of the tap, thus leading to a shift in the asynchrony). However, she was unable to demonstrate any systematic dependency of the size of the asynchrony on tap duration.
} 
A number of tapping studies have focused on the influence of the interval duration of the pacing signal on the size of the asynchrony. In general, the upper limit for still being able to synchronize with the pacing signal is about 2000 to $2500 \mathrm{~ms}$, whereas the lower limit is 200 to 300 ms (Eckert, 1992; Fraisse, 1966; Mates et al., 1992; Peters, 1989). The smallest interval to which people are able synchronize depends partly on limits within the motor system. The maximum tapping rate is in the order of 160 to 180 ms (Cousins, Corrow, Finn, \& Salamone, 1998; Shimoyama, Ninchoij, \& Uemura, 1990). The upper limit reveals itself in a performance switch. When the interval is too long to anticipate the next signal, participants tend to react to the signal rather than synchronize. This may well indicate the upper limit of an internal timing mechanism responsible for the estimation of intervals (Mates, 1994b).

Several studies report an increase in negative asynchrony with increasing interval duration (Fraisse, 1966; Kolers \& Brewster, 1985; Mates et al., 1994; Miedreich, 2000; Peters, 1989; Pressing, 1998). This finding has often been cited as a refutation of the processing hypotheses described above. The processing of a single event (click or tap) should be independent of the interval between these events. However, Gehrke (1996) identified the tap force as a possible intervening variable. The idea was that tapping at a faster rate (short intervals) is accompanied by more forceful taps than tapping at a slow rate. Forceful taps, however, lead to reduced asynchronies, which could explain the dependency of the asynchrony on the interval duration. Gehrke (1996; Experiment 3) trained participants to produce taps with the same amount of force in conditions with different interval durations (between 600 and $1100 \mathrm{~ms}$ ). As expected, the dependency of the asynchrony on the length of the interstimulus interval disappeared under these conditions, thus supporting Gehrke's interpretation of the effect.

In typical synchronization experiments, the interval between successive stimuli is empty. This is completely different from the situation in music, in which the intervals between two beats are filled with a number of tones. Thaut, Rathbun, and Miller (1997) tested the idea that asynchrony might depend on this factor. They asked participants to tap not only in synchrony with a simple metronome but also to a piece of music as well, and observed a reduction in the amount of asynchrony under the latter condition. $^{3}$

This result was replicated and extended by Wohlschläger and Koch (2000). They introduced a condition in which the tones between the beats were presented randomly (instead of rhythmically). This finding suggests the interpretation that it is not music but, more generally, the structure of the interval between the pacing signals that is the important factor reducing the asynchrony. Their corresponding hypothesis is based on the assumption that the asynchrony is an error in time estimation that occurs only in empty intervals. When the interval between two tones is empty, the duration of the interval is underestimated, and the corresponding tap is produced too soon.

\section{Asymmetrical Error Tolerance}

In almost all studies described above, the dependent variable was the mean asynchrony of taps within a longer sequence. However, any study of the evaluation and control processes that are the precondition for a stable tapping performance requires an analysis of the sequential dependencies within a sequence of taps. Repp (2000) introduced small shifts of the interstimulus interval (e.g., a single 20-ms reduction of the interval). He then analyzed whether and how participants corrected these artificial

${ }^{3}$ But see Repp (personal communication on comparing Experiments 1 and 3 in Repp, 2000), who was unable to replicate this difference. 
errors. Results indicated that they were corrected immediately within the next two taps. In addition, Repp (2000) was able to demonstrate that even shifts as small as $4 \mathrm{~ms}$ were corrected, indicating that this error correction is even possible for shifts below the perceptual threshold. In contrast to the usual assumption that the limits in perceptual processing determine corrections in the motor system (see, e.g., Mates 1994a, 1994b), he concludes that the motor system has access to temporal information at a more precise level than the perceptual system.

Another possible way of studying evaluation processes is to perform separate analyses of positive and negative asynchronies and the corresponding next tap. These analyses seem to indicate a different correction mechanism for positive and negative asynchronies. When the tap follows the click (positive asynchrony), this asynchrony is corrected immediately. However, this might not be true for negative asynchronies in which the critical interval within which an asynchrony is tolerated by the system seems to be at least $60 \mathrm{~ms}$ (Müller, Aschersleben, Koch, Freund, \& Prinz, 1999). Although this finding is based on a reanalysis of existing data, it is supported by a perceptual experiment in which participants had to indicate whether they perceived an asynchrony between tap and click (Koch, 1999). Once again, an asymmetry was observed: Participants were more sensitive to positive than to negative asynchronies. Further support comes from brain imaging studies. Using MEG measurement, Müller et al. (2000) were able to demonstrate that brain activity differs as a function of whether it is correlated with positive or negative asynchronies.

One possible interpretation of the different processing of positive and negative asynchronies is the hypothesis that the cognitive system prefers a situation in which the short click event is embedded in the long tap event. Such a strategy would avoid the gaps between events that would always arise in such situations in which the tap onset takes place after click offset (gap avoidance, Müller et al., 1999). This general idea is supported by experiments in which additional auditory feedback is presented before tap onset. Whereas under conditions with delayed auditory feedback (in which the feedback falls within the tap duration), an integration of the different feedback components can be observed (see first part of this article), something completely different happens under conditions with early feedback (when the feedback is presented before the tap onset). Under these conditions, no influence of the feedback on the timing of the tap can be observed, thus, no integration takes place (Mates \& Aschersleben, 2000).

\section{CONCLUSIONS}

The aim of this article has been to summarize evidence on the timing of simple movements (with the synchronization paradigm as a typical example). After describing the paradigm and a typical effect — negative asynchrony — we have discussed one class of accounts in more detail: the representational models. These models assume that synchrony is established at the level of central representations, and that the tap is represented by its sensory feedback. Negative asynchrony arises as a consequence of differences in peripheral and/or central processing times. Evidence in favor of these models is based mainly on the manipulation of the temporal distance between the tap and its central representation.

The second part of this article reports further evidence from the synchronization paradigm and additional accounts. These studies are based mainly on manipulations of the features of the pacing signal and cannot be explained easily by the representational models. On the other hand, the different additional accounts developed to explain these empirical findings are also unable to account for all the available findings. 
At first sight, this might be a problem; however, a closer look at the different accounts makes it clear that they are not as mutually exclusive as the discussion in the current literature might suggest. For example, it seems reasonable to assume that when confronted with temporally extended stimuli (like syllables or tones), the point in time that the cognitive system uses to synchronize with the tap is not the onset but somewhere within the stimulus (as the p-center hypothesis assumes). At the same time, this does not exclude the possibility that the tap is represented by its sensory feedback, and that representations of click and tap (which are not determined by their onsets) are synchronized. One can even go one step further and state that the sensory accumulator model proposed by Gehrke and colleagues (Aschersleben et al., in press a; Gehrke, 1996) is a specific version of the p-center account. This is because the sensory accumulator model assumes that representations of clicks and taps unfold in time and cannot be considered as point-like events. The peaks at which the corresponding accumulation functions reach their respective thresholds determine the times at which the two events are perceived-the perceptual center. Thus, it seems to be the perceptual center of an (perceptual or motor) event that participants use as the reference point for synchronization. Moreover, this perceptual center is determined not only by stimulus length or stimulus intensity in the perceptual events but also by factors such as the intensity of somatosensory feedback in the motor events.

On top of such sensorily determined, presumably automatic processes, there may also be a level of "strategic" components influencing the asynchrony. The idea of gap avoidance (with the system preferring a short click embedded in a long tap) suggested by Müller et al. (1999) can be combined easily with the idea that the costs for correcting positive and negative errors might differ (Miedreich, 2000). Correcting negative asynchronies is relatively easy, because it means lengthening the interval between two consecutive taps. However, correcting positive asynchronies would necessitate shortening the interval, which is consistent with a loss of processing time.

In sum, it seems reasonable to call for integrative accounts that try to combine different hypotheses (as suggested in the last two paragraphs) instead of a continuation of research that focuses mainly on pitting different hypotheses against each other.

\section{REFERENCES}

Aschersleben, G. (1994). Afferente Informationen und die Synchronisation von Ereignissen [Afferent information and the synchronization of events]. Frankfurt, Germany: Lang.

Aschersleben, G. (2000a). Effects of training on the timing of simple repetitive movements. Manuscript submitted for publication.

Aschersleben, G. (2000b). Knowledge of results and the timing of actions (Paper No. 1/2000). Munich: Max Planck Institute for Psychological Research.

Aschersleben, G., Gehrke, J., \& Prinz, W. (2001). Tapping with peripheral nerve block: A role for tactile feedback in the timing of movements. Experimental Brain Research, 136, 331-339.

Aschersleben, G., Gehrke, J., \& Prinz, W. (in press a). A psychophysical approach to action timing. In C. Kaernbach, E. Schröger, \& H. Müller (Eds.), Psychophysics beyond sensation: Laws and invariants in human cognition. Hillsdale, NJ: Erlbaum.

Aschersleben, G., \& Prinz, W. (1995). Synchronizing actions with events: The role of sensory information. Perception \& Psychophysics, 57, 305-317.

Aschersleben, G., \& Prinz, W. (1997). Delayed auditory feedback in synchronization. Journal of Motor Behavior, 29, 35-46.

Aschersleben, G., Stenneken, P., Cole, J., \& Prinz, W. (in press b). Timing mechanisms in sensorimotor synchronization. In W. Prinz \& B. Hommel (Eds.), Attention and performance. XIX. Common mechanisms in perception and action. Oxford: Oxford University Press.

Auxiette, C. (1992). Coordination et synchronisation de deux sequences sonores percues et produites 
[Coordination and synchronization of two perceived and produced auditory sequences]. Unpublished doctoral dissertation, Université René Descartes, Paris.

Auxiette, C., \& Boucart, M. (1995). The role of perception in synchronization tasks. Proceedings of the 36th Annual Meeting of the Psychonomic Society (p. 32), Los Angeles.

Bard, C., Paillard, J., Lajoie, Y., Fleury, M., Teasdale, N., Forget, R., \& Lamarre, Y. (1992). Role of the afferent information in the timing of motor commands: A comparative study with a deafferent patient. Neuropsychologia, 30, 201-206.

Bard, C., Paillard, J., Teasdale, N., Fleury, M., \& Lajoie, Y. (1991). Self-induced versus reactive triggering of synchronous hand and heel movement in young and old subjects. In J. Requin \& G. E. Stelmach (Eds.), Tutorials in motor neuroscience (pp. 189-196). Amsterdam: Kluwer.

Billon, M., Bard, C., Fleury, M., Blouin, J., \& Teasdale, N. (1996). Simultaneity of two effectors in synchronization with a periodic external signal. Human Movement Science, 15, 25-38.

Cole, J. D., \& Sedgwick, E. M. (1992). The perceptions of force and of movement in a man without large myelinated sensory afferents below the neck. Journal of Physiology, 449, 503-515.

Cooke, J. D., Brown, S., Forget, R., \& Lamarre, Y. (1985). Initial agonist burst duration changes with movement amplitude in a deafferented patient. Experimental Brain Research, 60, 184-187.

Cousins, M. S., Corrow, C., Finn, M., \& Salamone, J. D. (1998). Temporal measures of human finger tapping: Effects of age. Pharmacology, Biochemistry \& Behavior, 59, 445-449.

Drewing, K., \& Aschersleben, G. (2001). Reduced timing variability during bimanual coupling: A role for sensory information. Manuscript submitted for publication.

Dunlap, K. (1910). Reactions on rhythmic stimuli, with attempt to synchronize. Psychological Review, 17, 399-416.

Eckert, P. (1992). Auswirkungen der Intervallänge und der Rückmeldung auf eine sensumotorische Synchronisationsaufgabe [Influence of interstimulus interval duration and feedback on a sensorimotor synchronization task] (Paper No. 8/92). Munich: Max Planck Institute for Psychological Research.

Fraisse, P. (1966). L'anticipation de stimulus rythmiques, vitesse d'établissement et précision de la synchronisation [Anticipation of rhythmical stimuli, set-up speed and accuracy of synchronization]. L’Année Psychologique, 66, 15-36.

Fraisse, P. (1980). Les synchronisations sensori-motrices aux rythmes [The sensorimotor synchronization of rhythms]. In J. Requin (Ed.), Anticipation et comportement (pp. 233-257). Paris: Centre National.

Fraisse, P., Oléron, G., \& Paillard, J. (1958). Sur les repères sensoriels qui permettent de contrôler les mouvements d'accompagnement de stimuli périodiques [On the sensory reference points that allow for controlling movements accompanying periodic stimuli]. L'Année Psychologique, 58, 322-338.

Fraisse, P., \& Voillaume, C. (1971). Les repères du sujet dans la synchronisation et dans la pseudosynchronisation [Subject's reference point during synchronization and pseudo-synchronization]. L'Année Psychologique, 71, 359-369.

Gehrke, J. (1995). Sensorimotor synchronization: The intensity of afferent feedback affects the timing of movements (Paper No. 15/1995). Munich: Max Planck Institute for Psychological Research.

Gehrke, J. (1996). Afferente Informationsverarbeitung und die Synchronisation von Ereignissen [Afferent information processing and the synchronization of events]. Unpublished doctoral dissertation, Ludwig Maximilians University, Munich.

Johnson, W. S. (1898). Researches in practice and habit. Studies from the Yale Psychology Laboratory, 6, 51-105.

Koch, R. (1999). Detection of asynchrony between click and tap (Paper No. 1/1999). Munich: Max Planck Institute for Psychological Research.

Kolers, P. A., \& Brewster, J. M. (1985). Rhythms and responses. Journal of Experimental Psychology: Human Perception and Performance, 11, 150-167.

Ludwig, C. (1992). Experiment zur Synchronisation akustischer Führungssignale [Experiment on the synchronization of auditory pacing signals]. Unpublished term paper, Ludwig Maximilians University, Munich.

Mates, J. (1994a). A model of synchronization of motor acts to a stimulus sequence. II. Stability analysis, error estimation and simulations. Biological Cybernetics, 70, 475-484.

Mates, J. (1994b). A model of synchronization of motor acts to a stimulus sequence. I. Timing and error corrections. Biological Cybernetics, 70, 463-473.

Mates, J., \& Aschersleben, G. (2000). Sensorimotor synchronization: The influence of temporally displaced auditory feedback. Acta Psychologica, 104, 29-44. 
Mates, J., Müller, U., Radil, T., \& Pöppel, E. (1994). Temporal integration in sensorimotor synchronization. Journal of Cognitive Neuroscience, 6, 332-340.

Mates, J., Radil, T., \& Pöppel, E. (1992). Cooperative tapping: Time control under different feedback conditions. Perception \& Psychophysics, 52, 691-704.

Miedreich, F. (2000). Zeitliche Steuerung von Handlungen. Empirischer Test des Wing-Kristofferson Modells [Timing of actions. Empirical test of the Wing-Kristofferson model]. Aachen, Germany: Shaker.

Miyake, I. (1902). Researches on rhythmic action. Studies from the Yale Psychology Laboratory, 10, $1-48$.

Morton, J., Marcus, S. M., \& Frankish, C. (1976). Perceptual centers (P-centers). Psychological Review, 83, 405-408.

Müller, K., Aschersleben, G., Koch, R., Freund, H.-J., \& Prinz, W. (1999). Action timing in an isochronous tapping task: Evidence from behavioral studies and neuroimaging. In G. Aschersleben, T. Bachmann, \& J. Müsseler (Eds.), Cognitive contributions to the perception of spatial and temporal events (pp. 233-250). Amsterdam: Elsevier.

Müller, K., Schmitz, F., Schnitzler, A., Freund, H.-J., Aschersleben, G., \& Prinz, W. (2000). Neuromagnetic correlates of sensorimotor synchronization. Journal of Cognitive Neuroscience, 12, $1-10$.

Müller, K., Aschersleben, G., Schmitz, F., Schnitzler, A., Freund, H.-J., \& Prinz, W. (2001). Modalityspecific central control units in sensorimotor synchronization. Manuscript submitted for publication.

O'Boyle, D. J., \& Clarke, V. L. (1996). On the source of the negative synchronization error during temporal-tracking performance. Brain Research Association Abstracts, 13, 40.

Paillard, J. (1949). Quelques données psychophysiologiques relatives au déclenchement de la commande motrice [Some psychophysiological data relating to the triggering of motor commands]. L'Année Psychologique, 48, 28-47.

Peters, M. (1989). The relationship between variability of intertap intervals and interval duration. Psychological Research, 51, 38-42.

Pressing, J. (1998). Error correction processes in temporal pattern production. Journal of Mathematical Psychology, 42, 63-101.

Prinz, W. (1990). A common coding approach to perception and action. In O. Neumann \& W. Prinz (Eds.), Relationships between perception and action: Current approaches (pp. 167-201). Berlin: Springer.

Prinz, W. (1992). Why don't we perceive our brain states? European Journal of Cognitive Psychology, 4, 1-20.

Prinz, W. (1997). Perception and action planning. European Journal of Cognitive Psychology, 9, 129154.

Repp, B. H. (1999). Control of expressive and metronomic timing in pianists. Journal of Motor Behavior, 31, 145-164.

Repp, B. H. (2000). Compensation for subliminal timing perturbations in perceptual-motor synchronization. Psychological Research, 63, 106-128.

Shimoyama, I., Ninchoij, T., \& Uemura, K. (1990). The finger-tapping test. Archives of Neurology, 47, 681-684.

Stenneken, P., Aschersleben, G., Cole, J., \& Prinz, W. (in press). Self-induced versus reactive triggering of synchronous movements. A comparative study with a deafferented patient. Psychological Research.

Stevens, L. T. (1886). On the time sense. Mind, 11, 393-404.

Thaut, M. H., Rathbun, J. A., \& Miller, R. A. (1997). Music versus metronome timekeeper in a rhythmic motor task. International Journal of Arts Medicine, 5, 4-12.

Thaut, M. H., Tian, B., \& Azimi-Sadjadi, M. R. (1998). Rhythmic finger tapping to cosine-wave modulated metronome sequences: Evidence of subliminal entrainment. Human Movement Science, 17, 839-863.

Vorberg, D., \& Wing, A. M. (1996). Modeling variability and dependence in timing. In H. Heuer \& S. Keele (Eds.), Handbook of perception and action: Motor skills (pp. 181-262). London: Academic Press.

Vos, P. G., Helsper, E. L., \& van Kruysbergen, N. (1992). Tracking simple rhythms under pseudosynchronization conditions. Proceedings of the Fourth Rhythm Workshop: Rhythm perception and production (pp. 37-42). Bourges, France. 
Vos, P. G., Mates, J., \& Kruysbergen, N. W., van (1995). The perceptual centre of a stimulus as the cue for synchronization to a metronome. The Quarterly Journal of Experimental Psychology, 48, 1024-1040.

Wohlschläger, A., \& Koch, R. (2000). Synchronization error: An error in time perception. In P. Desain \& L. Windsor (Eds.), Rhythm perception and production (pp. 115-127). Lisse, The Netherlands: Swets \& Zeitlinger. 